\title{
COMMUNITY BASED MATERNAL AND CHILD HEALTH CARE IN NEPAL : SELF- REPORTED PERFORMANCE OF MATERNAL AND CHILD HEALTH WORKERS
}

\author{
Chhetry $S^{*}$, Clapham S*, Basnett I** \\ * Save the Children/US, ** DFID Nepal, *** Nepal Safer Motherhood Project
}

\begin{abstract}
The performance of a sample of $\mathbf{1 1 2}$ refresher trained Maternal and Child Health Workers (MCHW) was assessed over a nine-month period, using a self-reporting questionnaire. The findings show that the MCHWs are providing obstetric services, including antenatal care, birth attendance and postnatal and newborn care, at community level and identifying complications for referral, but their productivity levels are very low, particularly in the remote hill areas, where they are most needed. In order to increase their effectiveness, greater emphasis needs to be placed on the creation of an enabling environment, both in terms of professional support and recognition at community level. This requires more technical and logistical backup and the promotion of greater awareness amongst women and their families about the importance of midwifery care and skilled birth attendance.
\end{abstract}

Key Words: Skilled attendance, enabling environment, performance.

\section{INTRODUCTION}

Maternal and Child Health Workers (MCHW) were first introduced in 1993, based at Sub Health Posts (SHP) to serve the maternal and child health needs of communities. They are usually local women, who receive a three-month basic training, including theory and supervised clinical practice in an assigned hospital. In 1999, a six-week "refresher training” for MCHWs was piloted, to upgrade their skills to an acceptable level for village-based skilled birth attendants, and an Essential Obstetric Care (EOC) kit box was supplied to each participant for attending home births. In 2000, as part of the national effort to reduce maternal mortality, the Ministry of Health initiated a strategy promoting community based skilled birth attendance ${ }^{1}$ to complement the strategy initiated in 1998 to increase access to EOC services. ${ }^{2}$ This included extending the MCHW refresher training to increase the number of skilled attendants available in communities. As the training covers additional material to that in the basic curriculum, it is not technically refresher in nature, but constitutes an upgrading of skills and knowledge. However, as it is commonly referred to as refresher training, the term will be used in this paper, which reviews the findings from a self-reported performance assessment of 112 refresher trained MCHWs.

\section{The Nepal Safer Motherhood Project (NSMP)}

The Nepal Safer Motherhood Project (NSMP) was initiated in 1997, as a joint venture between the Ministry of Health and the Department for International Development (DFID), with the purpose of increasing utilisation of quality midwifery and EOC services. It is based on two complementary components: service provision (coverage and quality of EOC); and access (social and behavioural issues affecting utilisation of services).

Address for correspondence :

Dr. Indira Basnett

Nepal Safer Motherhood Project, Teku

Email: indira@ssmp.org.np

Received Date : $9^{\text {th }}$ October, 2004

Accepted Date : 15 th June, 2005 
Currently NSMP works in ten districts, providing support at facility and community level, as well as providing input to policy level discussions, including the emerging skilled attendance debate.

\section{The Skilled Attendance Debate in Nepal}

One of the key international learning points for safe motherhood during the last decade is that,

"Having a health worker with midwifery skills present at childbirth, backed up by transport in case emergency referral is required, is perhaps the most critical intervention for making motherhood safer". 3

Internationally, there has been increasing interest in the role of skilled attendants in reducing maternal mortality. Graham ${ }^{4}$ estimates that around $16 \%$ to $33 \%$ of all maternal deaths due to the four main causes (obstructed labour, haemorrhage, sepsis and eclampsia) could be prevented by skilled attendance at birth. In November 2000 the Safe Motherhood Inter Agency Group (IAG) hosted an international conference on this topic, in which a team from Nepal participated. The conference presented evidence of the effectiveness of a skilled attendance strategy in reducing maternal mortality, and important facets of this, with a particular emphasis on the need for an "enabling environment" for health workers. ${ }^{5}$

At national level, the Maternal Mortality and Morbidity Study (MMMS) ${ }^{6}$ was influential in highlighting the high proportion (46.3\%) of deaths resulting from postpartum haemorrhage $(\mathrm{PPH})$, with or without retained placenta. Given that $89 \%$ of births take place at home ${ }^{7}$ and that, on average a woman will die within two hours of onset of $\mathrm{PPH},{ }^{8}$ for many Nepali women there is no time to reach facility based EOC services. This means that community based obstetric first aid is critical. Although skilled attendance normally includes both facility and home based care, in Nepal, because of the difficulties of travel, the term has largely been identified with community/ home-based care. MCHWs were chosen as the focus for efforts to increase skilled attendance because they are local women, acceptable to their communities, and have primary responsibility for provision of maternal and neonatal health care at community level.

The key strategic issue facing the Ministry of Health was whether MCHWs could achieve the level required to be termed skilled birth attendants, and thus play a part in reducing maternal mortality. This meant matching the skill and competency level expected from an MCHW after refresher training with international definitions for a skilled attendant. However, although these specify a set of required skills, the level of competence for each skill is not clear. For example it is stated that a skilled attendant must have life saving skills for dealing with PPH, but does not specify the skill level which can range from rubbing up a contraction or manual compression. ${ }^{9}$ The IAG has acknowledged that international agreement on this issue is needed, particularly when dealing with staff trained to a relatively basic level, such as MCHWs. The IAG review ${ }^{9}$ states "lower cadres may have some of these skills, particularly for the management of normal labour and delivery, but further discussion and consensus is needed on the most appropriate role for them in the management of complications and in a skilled attendance strategy". Thus in countries such as Nepal where lower cadres of staff, such as MCHWs, are a key means of delivering safe motherhood services in rural areas, their role in a skilled attendance strategy remains unclear. Recent literature ${ }^{10}$ is beginning to assist with more detail about required competency levels, but much remains to be done in this area.

Three key questions faced the Ministry of Health in 2000 to 2001:

- Given the lack of international clarity about the level of competence required for a skilled attendant, what was the minimum competency level required to impact on maternal mortality?

- To what skill and competency level would it be possible to train and sustain MCHWs?

- Would that skill and competency level make a difference to maternal mortalities?

\section{Piloting MCHWS as Skilled Attendants}

The four components of the Nepal skilled attendance strategy are:

- $\quad$ Upgrading the technical skills of MCHWs in midwifery and obstetric first aid

- Developing an enabling environment for MCHWs at their SHP

- $\quad$ Setting up systems to ensure that essential supplies and equipment are available at the SHP

- Developing effective working relationships between MCHWs, district hospitals and District Public Health Office (DPHO) staff.

The strategy was piloted for three years (1999 to 2002), led by the Family Health Division (FHD) of the Department of Health Services (DoHS), with support from NSMP, the USAID funded Maternal and Neonatal Health (MNH) programme and UNFPA. NSMP supported implementation in project districts by providing on-site monitoring, and working to develop an enabling environment at the SHPs, MNH focused on training curriculum development, and UNFPA provided the EOC kit box for MCHWs. 
Training: The National Health Training Centre (NHTC) and FHD developed the competency-based six-week refresher training curriculum for MCHWs, with technical assistance from safe motherhood stakeholders. This was set at a level considered realistic for MCHWs, to upgrade their knowledge and develop their clinical competency to provide quality midwifery and newborn care, identify obstetric complications, provide obstetric first aid and make timely referrals to an appropriate facility.

The enabling environment: The experience of NSMP is that training alone does not bring real change without a supportive working environment. ${ }^{11}$ As a first step to ensuring support after the MCHW refresher training, the District Health Office (DHO) organises a two-day “enabling environment” workshop, which includes the SHP In-charge, Village Development Committee (VDC) Secretary and MCHW. The objective is to orient the VDC and SHP teams about the MCHW's job, to motivate them to create an enabling environment to help her fulfil her job description, and to inform the community about her new skills. The roles of the SHP and community in creating a supportive environment are discussed, and each SHP team is asked to develop a work plan to this effect for the next six months, including promoting the MCHW as an skilled birth attendant in the community, developing local mechanisms for replenishing the drugs/ supplies, ensuring adequate infrastructure for service provision, and providing incentives for MCHWs to provide home-based maternity services (the amount to be set by individual VDCs).

The DHO organises six-monthly review meetings with the SHP and VDC team to assess progress against their plan, and discuss the midwifery services provided, types of obstetric complications managed, referrals made and practical problems MCHWs have encountered.

In 2003 a national assessment of the performance of MCHWs was carried out, ${ }^{12}$ which looked at both the clinical competency levels and working environment of MCHWs. It concluded that the refresher training was successful in upgrading the skills of MCHWs to provide basic obstetric first aid, and recommended extending it to all MCHWs. However, the low productivity of MCHWs was also noted as a key issue, and the need for more support at facility and community level to enable them to fulfil their potential as service providers.

\section{The Study}

The study group comprised 222 MCHWs in nine NSMP supported districts, all of whom had received refresher training at the time when the study was carried out.

\section{Study rationale and objectives}

NSMP originally planned to conduct an onsite follow-up for all refresher trained MCHWs, to assess the extent to which they were implementing their new skills and knowledge, and to provide support and coaching. However, this proved impractical because of the limited numbers of nurse trainers available to do follow up and the armed conflict, which restricted travel by outsiders to affected areas. As a substitute, a self-assessment form for MCHWs to complete was developed, in consultation with FHD, NHTC and the concerned DHOs.

The objectives of the assessment were to:

- $\quad$ Assess the range of midwifery and obstetric care services provided by MCHWs post refresher training

- Determine the effectiveness of replenishment mechanisms for drugs and supplies in the EOC kit box

- Identify the problems and issues related to provision of services

The questionnaire focused on the key skills specified in the refresher training curriculum, including provision of antenatal (ANC), intrapartum and postnatal services, newborn care and obstetric first aid and referral to higher facilities. MCHWs were also asked whether they had been replenishing the contents of the kit box, if they had encountered problems in delivering services at the SHP and the approaches used to overcome them.

\section{METHODOLOGY}

The assessment covered a nine-month period from July 2001 to April 2002. The questionnaire forms were sent to all 222 refresher trained MCHWs, through the DHOs, including instructions for completion and submission.

\section{Study Limitations}

Out of the total 222 sample MCHWs, only 112 correctly completed the forms for the whole nine-month period, and therefore only 112 forms were included in the analysis.

As the study only covered refresher trained MCHWs, it is not possible to make a comparison between the performance of refresher and non-refresher trained MCHWs. However the findings of the national MCHW assessment ${ }^{12}$ indicate that the training does have a significant effect on performance, both in skill competency and productivity.

Initially an attempt was made to establish a baseline for comparison through inclusion of pre-training data in the questionnaire. However, this proved impossible because of poor record keeping, and misplacement of records at the SHP. It was therefore not possible to compare the performance of the MCHWs pre and post-training. 
Table I : Services Provided by the 112 MCHWs over the Nine-Month Period of the Study

\begin{tabular}{lcc}
\hline \multicolumn{1}{c}{ Services Provided } & Total Number for all MCHWs & Avg per MCHW/Month \\
\hline ANC checkups & 15,193 & 15 \\
Referrals for ANC & 356 & 0.4 \\
Births attended & 1,668 & 1.6 \\
Prior referrals for birth care & 247 & 0.2 \\
Postnatal care & 4,317 & 4.2 \\
Birth complications managed and/or referred & 900 & 0.9 \\
Newborn care & 1,668 & 1.6 \\
Newborn complications managed and/or referred & 549 & 0.5 \\
\hline
\end{tabular}

\section{Findings}

\section{Services provided}

The table I shows total figures for the services provided by the 112 MCHWs over the period of the assessment, including antenatal care, birth attendance, postnatal and newborn care, and referrals.

Antenatal care: The average number of pregnant women seen by the sample MCHWs is 15 per month per MCHW, with a range of 1.7 to 27.6, including both first and subsequent visits. This is comparable with the findings of the national assessment, ${ }^{12}$ which showed an average of 17 ANC checkups per MCHW per month. Based on the expected numbers of pregnancies in the sample areas, the study found that on average $12.3 \%$ of pregnant women were receiving ANC services from the MCHWs, higher than the DHS 2001 figures, ${ }^{7}$ which showed only 3\% of mothers receiving antenatal care from an MCHW. The study percentages were slightly higher in the three terai districts, at $14.7 \%$, compared with $7.2 \%$ in the hill/mountain districts.

Out of the 15,193 antenatal encounters, only 356 (2.3\%) were referred to EOC facilities for further management, a surprisingly low figure. The most common reasons for referral were anaemia (37.6\%), P/V bleeding (22.1\%) and abnormal lie (12.9\%). Anaemia was diagnosed on the basis of signs and symptoms stated in the reproductive health clinical protocol only, since laboratory facilities are not available at SHP level.

Birth attendance: On average the MCHWs attended 1.6 births per month each. All were home births, as is the cultural norm in Nepal. The number is slightly higher in terai, at 1.95 per month, than in hill /mountain districts. ${ }^{1,12}$ This low productivity is confirmed by the national assessment, ${ }^{12}$ which records an average of 1.5 births per month per refresher trained MCHW. The percentage of expected births attended by the MCHWs was very low, only $1.4 \%$ of expected births were attended by MCHWs. This figure is comparable with the DHS 2001 figure $^{7}$ of less than $1 \%$ of births managed by MCHWs.
The MCHWs referred 247 cases for management of the birth at a higher facility, mainly because of prolonged labour (42.5\%) or abnormal lie (42.9\%). This accounts for $15 \%$ of the 1,669 births they attended, and is in line with the WHO estimation that $15 \%$ of all pregnancies may develop serious complications and need referral. ${ }^{13}$

Postnatal care and obstetric first aid: On average the MCHWs provided postnatal care for 4.2 women per month, including following up women they had assisted during the birth, for whom they felt accountable and involved, and call-outs to other women with postpartum complications. The number includes first (within 6 hours of birth), and subsequent visits at 6 days and 6 weeks. This is in line with the national assessment, ${ }^{11}$ which shows a range of 0.6 to 10.4 post natal checkups per month per MCHW, and supports the 2001 DHS $^{7}$ finding that postnatal care is considered a low priority, and less than one in five postnatal women receive a check up from any health staff.

On average the MCHWs reported managing less than one postpartum obstetric complication per month (with a range of 0.4 to 1.2), including emergency cases for which they were called, in addition to the births they attended. Puerperal sepsis (32.2\%) and retained placenta (31.8\%) were the most common conditions, and the percentage of sepsis cases is higher than that found in the MMMS (11.8\% of total complications), which may be because the MCHWs recorded all cases handled immediately, whereas the MMMS relied on memory over a longer period. The MCHWs appear confident and competent in identifying infections, treating with antibiotics and referring to an appropriate facility. They were able to manage retained placenta with controlled cord traction, but for adherent placenta they needed to refer to a higher facility. PPH cases accounted for $18.7 \%$ of the total number of complications handled, but the numbers for individual MCHWs were again very low, ranging from 0 to 3.4 over the nine-month period, again supporting the findings of the national assessment.

Newborn care: The MCHWs provided routine newborn care only in cases where they attended the birth. This included dry- 
ing the baby, wiping the nose and eyes, providing suction for the airways if needed, cutting the cord and encouraging immediate breastfeeding. No neonatal deaths were reported during the study period.

The MCHWs reported managing and/or referring a total of 549 newborn complications ( 0.5 per MCHW per month), including additional cases for which they were called, as well as those for which they attended the birth. The most common conditions reported were newborn infections (55.37\%) followed by birth asphyxia (30.05\%). This is in line with the findings of the Save the Children US Saving Newborn Lives (SNL) study of 2002,14 which show that neonatal infections and birth asphyxia are two leading causes of hospital admission and mortality for neonates.

\section{Enabling Environment}

The MCHWs reported the following barriers to providing midwifery and EOC services:

Physical facilities: The majority of the MCHWs reported that the SHPs do not have the minimum basic physical facilities needed even for private physical examination of pregnant women. They lack appropriate furniture, supplies, equipment and drugs for midwifery services, and there is no sterilising equipment. Some individuals also reported that registers were not available for recording the services provided.

EOC kit box: The RH clinical protocol states that two EOC kit boxes should be provided to each SHP, one for the MCHW to keep at home, and the other for the SHP, but only one kit box is provided at the end of the refresher training. Eight percent of the MCHWs also reported that items such as the suction bulb, BP set, and cord-cutting scissors were missing from kit boxes, and two had not even received a kit box at all.

Replenishment of supplies: One quarter of the MCHWs reported that there is no clear system for replenishment of kit box supplies or other drugs and equipment for their SHP, either from the DHO or local medical shops. They commented that the majority of women and their families were reluctant to pay for the drugs and supplies used, and even to purchase the sutkiri samagiri (Clean Delivery Kit) carried by the MCHW, as they believed these supplies came from the government free of cost, and therefore the MCHW was making a profit from their sale. They reported that this was particularly difficult if the client was poor. The SHP committees and VDCs had not fixed standard charges for call-out services such as birth attendance, managing PPH or retained placenta, which made it even more difficult for MCHWs to obtain any reimbursement for items used or their time.
Professional support: The MCHWs universally reported a lack of professional support.

Geographical terrain: The MCHWs reported difficulty in attending night calls because of lack of transport, particularly in hilly/mountain areas. They said they felt unsafe going out after dark, and in some conflict-affected districts it is not possible to travel at night.

Health seeking behaviour: Two percent of the MCHWs reported experiencing little trust from their communities, saying that people do not value their services or listen to them. They mentioned low levels of awareness among the women and their families about the need for care during pregnancy, delivery and the postpartum period, and the benefits of seeking help from skilled health personnel. It was said that many people still have more faith in traditional healers and Traditional Birth Attendants (TBA) and therefore seek help from them first.

Socio-economic factors: Poverty and illiteracy were identified as major barriers to people seeking help from trained MCHWs, because of the cost and a lack of understanding of the potential benefits. As a result, it was said that women often sought help only at a late stage after complications had developed and their condition had become serious.

Skill limitations: Two of the MCHWs reported lack of confidence/skill in administering intra-venous fluids, and others also mentioned difficulty in managing a breech delivery.

Other: Three MCHWs mentioned the lack of dress allowance as a de-motivating factor, and those from terai suggested that bicycles should be provided to enable them to attend home births more easily.

\section{DISCUSSION AND CONCLUSIONS}

\section{What MCHWs are doing}

It is encouraging to note that the sample MCHWs appear to be competently identifying obstetric complications, providing first aid and referring to appropriate facilities. The figures show that the percentage of complications referred from the births attended (14.8\%) is in line with the WHO estimate that $15 \%$ of births will develop complications requiring a higher level of care, ${ }^{13}$ indicating the valuable referral role that MCHWs can play in saving women's lives at the time of childbirth. The sample MCHWs appear to be managing and referring newborns with complications (averaging 0.5 per MCHW per month), and since this figure is around one third of all the newborn care provided, this indicates that families are calling the MCHW for help if the baby is in difficulty. It is noticeable that the figures for infection are high (52\% of all complications), presumably as a 
result of the unhygienic conditions under which many home births take place. MCHWs are also called out for postpartum obstetric complications, such as puerperal sepsis, PPH and retained placenta, even when they have not been asked to attend the birth. Thus it appears that at least a proportion of families believe the MCHW can help in an emergency.

Although the figures are low, the sample MCHWs are providing ANC services for a significant number of women within their communities, averaging 15 women per MCHW per month. This is supported by the figures in the 2003 annual health management information service data, ${ }^{15}$ which show an average of 17 ANC checkups per month per MCHW. The sample MCHWs were found to be reaching an average of $12.3 \%$ of expected pregnant women in the community, more in the terai districts (14.7\%) than the hill/mountain areas (7.2\%), presumably as a result of better transport facilities and the fact that the higher population densities and higher levels of education make "word of mouth" more effective in promoting the benefits of ANC. These figures reflect increasing acceptance among communities of the value of at least one ANC check up, although anecdotal evidence suggests that only a very small percentage of women go more than once, indicating potential for further increase with appropriate public education and information measures.

\section{What MCHWs are not doing}

A key finding from this assessment, which supports the findings of other similar work, such as the 2001 DHS, ${ }^{7}$ the national MCHW Assessment ${ }^{12}$ and the annual health management information service data ${ }^{15}$ is that the overall productivity of MCHWs in the key areas of ANC services, birth attendance, postpartum and neonatal care is very low, particularly for birth attendance and postpartum care, as a proportion of the population covered and the expected number of pregnancies.

The number of women referred to higher facilities for ANC is also very low (2.3\% of total cases examined), compared with the WHO estimate of $15 \%$ of all pregnancies expected to develop complications, ${ }^{13}$ and thus it appears that many of these are not being picked up.

The percentage of expected births attended by the MCHWs was very low, at $1.4 \%$ of expected pregnancies, only slightly higher than the 2001 DHS data, ${ }^{7}$ which shows that less than $1 \%$ of births are attended by MCHWs. Similarly, only 3.5\% of the expected pregnant women received postnatal care from the sample MCHWs. Since the figures include visits immediately after the birth and within 6 weeks of the birth, it seems likely that the reality is even lower, reflecting the low priority given to postnatal care. A further consequence of the low number of births attended by the MCHWs is a correspondingly low figure for newborn care.

Attendance by skilled personnel at birth is crucial, both for sake of managing a normal birth safely and identifying complications early enough for referral to an appropriate facility after stabilising the condition of the mother/newborn. This is a role that MCHWs appear to be capable of fulfilling, but their full potential for saving lives is a long way from being realised.

\section{The enabling environment}

It is generally accepted, and indicated by the findings of both the national assessment of 2003 and this study, that an enabling environment is critical for effective performance increased productivity of MCHWs, both at the SHP and in the community. The feedback of MCHWs highlights their frustrations at the lack of basic facilities at the SHP and logistical support, almost complete absence of professional support from the $\mathrm{D} / \mathrm{PHO}$, and lack of recognition for their services within the community. These issues are echoed in the national assessment, which also concludes that until they are addressed it is unlikely that the low productivity of MCHWs will increase significantly. Clear guidelines are needed for the establishment of a simple and effective system for replacement of used items in the EOC kit box and other supplies needed for maternal care at the SHP, and linkages should be established with Community Drug Programmes (CDP) to ensure regular availability of essential life saving drugs at reasonable cost.

Regular monitoring and supportive supervision from the $\mathrm{D} / \mathrm{PHO}$ is essential to promote acceptance within the community of the benefits of ANC, skilled attendance at birth and postnatal care, and to build the confidence of MCHWs in providing services. Once public acceptance and trust of services begins to increase, this is likely to lead to an increase in the confidence of MCHWs, feeding into a virtuous circle of further acceptance by the community. Regular review meetings at the D/PHO can give MCHWs an opportunity to discuss the services they provide, look at skill areas that need updating, and to ensure that D/ PHOs fully understand the issues. Such meetings help MCHWs to feel valued, especially in the light of the reality of limited professional support visits, and encourage the VDC and SHP in-charge to feel involved and responsible for providing support for MCH services.

Additional remuneration for services outside of normal hours, such as birth attendance, is needed to motivate MCHWs to provide call-out services. The VDC has an important role to play in promoting use of MCHW services, and setting charges for services outside normal hours. Further professional support is also needed, in the form of a system for regular updating of 
MCHW skills at the district hospital, especially if there is evidence of low performance. This would ensure that MCHWs have access to up to date information, have the opportunity to ask questions and see what happens to clients they refer, and feel professionally supported and valued as members of a team.

In conclusion, this study demonstrates the potential of MCHWs as community based midwives with basic obstetric first aid skills, but indicates that this is currently under fulfilled. The key to increasing their effectiveness and utilisation lies in developing a more enabling professional environment and raising the awareness of communities about the value of their services.

\section{REFERENCES}

1. Department of Health Services, His Majesty's Government of Nepal. Meeting the challenge of skilled attendance at delivery: Nepal's experiences. 2000.

2. Family Health Division, Department of Health Services, Ministry of Health, Nepal. Safe Motherhood Policy. 1998.

3. Starrs A. \& IAGSM.1998. The Safe Motherhood Action Agenda: priorities for the next decade, Report on the Safe Motherhood Technical Consultation, 18-23 October 1997, Colombo, Sri-Lanka. New York: InterAgency Group for Safe Motherhood.

4. Graham WJ, Bell JS, Bollough CHW. Can skilled attendance at delivery reduce maternal mortality in developing countries?' In studies in HSO\&P (17). 2001.
5. Clapham S, Basnet I. Report on Conference "Saving lives: skilled attendance at childbirth" Tunisia. NSMP/Options UK. 2000.

6. Family Health Division, Department of Health Services, Ministry of Health, Nepal. Maternal Mortality and Morbidity Study. 1998.

7. Family Health Division, Department of Health Services, Ministry of Health, Nepal. Nepal Demographic Health Survey. 2001.

8. Averting Maternal Death and Disability (AMDD) Programme. Workbook: Using the UN Process Indicators of Emergency Obstetric Services. (P.15). Columbia University, Mailman School of Public Health. 2003.

9. The Safe Motherhood Inter-Agency Group (IAG). Skilled Attendance at Delivery: A Review of the Evidence (draft). New York: Family Care International. 2000.

10. World Health Organisation. Global Action for Skilled Attendance for Pregnant Women. WHO Geneva. 2001.

11. Family Health Division, Department of Health Services, Ministry of Health, Nepal. Report on performance evaluation of graduates of post basic diploma in midwifery course, 2002.

12. Family Health Division, Department of Health Services, Ministry of Health, Nepal. An Assessment of the Performance of maternal and Child Health Workers in Nepal. 2003.

13. Joint WHO/UNFPA/UNICEF/World Bank Statement on Reduction of Maternal Mortality. WHO, Geneva. 1999.

14. Save the Children/ Saving Newborn Lives. State of the World's Newborns: Nepal. Save the Children/US, Kathmandu. 2002.

15. Department of Health Services, Ministry of Health, Nepal. Annual Report, 2002/2003. 